\title{
Barreras para la práctica de ejercicio físico en universitarios mexicanos deportistas y no deportistas
}

Barriers for the practice of physical exercise in Mexican university athletes and non-athletes

\author{
José René Blanco Ornelas \\ Universidad Autónoma de Chibuahua, México \\ jblanco@uach.mx
}

\section{Ma. Concepción Soto Valenzuela}

Universidad Autónoma de Chihuahua, México

masoto@uach.mx

Josué Nájera Longoria

Universidad Autónoma de Chibuahua, México

jnajera@uach.mx

\section{Fernando Mondaca Fernández \\ Universidad Autónoma de Chibuahua, México \\ fmondaca@uach.mx}

\author{
Perla Jannet Jurado García \\ Universidad Autónoma de Chibuahua, México \\ pjurado@uach.mx
}

\section{RESUMEN:}

El objetivo de esta investigación consistió determinar las diferencias y similitudes entre universitarios mexicanos que practican un deporte de manera regular con los que no lo hacen en cuanto a sus perfiles de barreras para la práctica de ejercicio físico. La muestra total fue de 1223 estudiantes universitarios de 18-36 años de edad; 593 de ellos practican algún deporte y participan regularmente en torneos o competencias deportivas. El abordaje adoptado en la investigación se enmarcó dentro de un enfoque cuantitativo con un diseño descriptivo tipo encuesta. Todos los participantes contestaron el Autoinforme de Barreras para la Práctica de Ejercicio Físico de Niñerola y colaboradores. Los resultados del análisis multivariante de la varianza, seguido por los análisis de varianza univariados, mostraron que los universitarios que no practican de manera regular algún deporte son quienes reportan mayor presencia de barreras en las cuatro dimensiones estudiadas (ansiedad física y social, fatiga o pereza, obligaciones y falta de tiempo e instalaciones). Las diferencias encontradas parecen indicar que los universitarios no deportistas perciben mayor probabilidad en la presencia de barreras para la práctica de ejercicio físico, dificultándoles la práctica de actividad física en mayor medida que a los que practican un deporte de manera regular.

Palabras clave: Actividad física, Diferencias de género, Barreras, Autopercepciones.

\begin{abstract}
:
The aim of this research was to determine the differences and similarities between Mexican university students who practice a sport on a regular basis and those who do not in terms of their profiles of barriers for practicing physical exercise. The total sample was of 1223 university students of 18-36 years of age; 593 of them practice some sport and participate regularly in tournaments or sports competitions. The research is framed within a quantitative approach with a survey descriptive design. All the participants answered the Barriers Self-Report for the Physical Exercise Practice of Niñerola and collaborators. Results from the multivariate analysis of variance, followed by the univariate variance analysis, showed university students who do not regularly practice a sport are those who report the greatest presence of barriers in the four dimensions studied (physical and social anxiety, fatigue or laziness, obligations and lack of time and facilities). The differences found seem to indicate that the non-athletic university students perceive greater probability in the presence of barriers to the practice of physical exercise, making it difficult for them to practice physical activity more than those who practice a sport on a regular basis.
\end{abstract}

KEYWORDS: Physical activity, Gender differences, Barriers, Self-perceptions. 


\section{INTRODUCCIÓN}

En la sociedad actual se asume la actividad física como variable esencial en la salud de las personas (Reiner, Niermann, Jekauc \& Wol, 2013). Además, los hábitos saludables adquiridos en edades tempranas resultan claves para obtener y mantener una salud óptima a lo largo de la vida (Longmuiremail, Colley, Wherley \& Tremblay, 2014). Sin embargo, existen numerosos hallazgos en relación a la inactividad física y la consecuente dificultad en la adquisición de estilos de vida activos en los jóvenes (Cuenca-García et al., 2014). Por su parte, los estudios con población adulta han demostrando que los niveles son aún menores que en edades escolares (Cocca, Liukkonen, Mayorga-Vega \& Viciana, 2014; Reiner et al., 2013). Debido a esta evidencia, la lucha contra el sedentarismo y el incremento de la actividad física entre los jóvenes es un importante reto de salud pública y una prioridad científica (Gillis et al., 2013).

Por otro lado, una serie de estudios han mostrado que la participación en ejercicio físico se relaciona con una imagen corporal positiva (Martin \& Bassett, 2012; Tucker \& Mortell, 1993; Williams \& Cash, 2001). Se ha encontrado también que las personas más activas tienen una actitud más positiva hacia su propio cuerpo que los sujetos sedentarios (Tornero \& Sierras, 2008; Urrutia, Azpillaga, de Cos \& Muñoz, 2010); que la actividad física y el deporte son medios para mejorar la salud del sujeto y prevenir la obesidad (Camacho, Fernández \& Rodríguez, 2006; Katzmarzyk \& Janssen, 2003; Kokkinos, 2012) y que estas prácticas tienen un efecto positivo sobre el aspecto físico y el placer relacionado con la consecución de éste (Alley, 1991). Por esto, se ha propuesto que la práctica de ejercicio físico se debería explotar además de como medio de protección de la salud, como ámbito en el que explorar el placer corporal, la diversión y el goce (Alley, 1991).

Las barreras percibidas por la población para la realización de actividad física, por tanto, adquieren enorme importancia y aportan los factores cruciales para incrementar la actividad física realizada. Entre estas barreras, la literatura previa ha mostrado que la falta de tiempo (Steptoe et al., 2002), la influencia social ligada a la imagen corporal y otros factores socioeconómicos (Bibiloni, Pich, Córdova, Pons \& Tur, 2012), o la falta de recursos (Serrano-Sanchez et al., 2011) entre otras, son diferentes barreras identificadas como causantes del nivel de sedentarismo en diferentes poblaciones.

En este sentido, cobra especial relevancia indagar si realizar actividad física de manera regular contribuye o no a percibir una mayor probabilidad en la presencia de barreras para la práctica de ejercicio físico, dificultando la práctica de actividad física.

En este estudio se intenta determinar las diferencias y similitudes entre universitarios mexicanos que practican un deporte de manera regular con los que no lo hacen en cuanto a la percepción de barreras para la práctica de ejercicio físico.

\section{Metodología}

\section{Participantes}

Participaron en el estudio 1223 todos alumnos y alumnas de las licenciaturas que se ofrecen en la Facultad de Ciencias de la Cultura Física de la Universidad Autónoma de Chihuahua. Donde 593 de ellos practican algún deporte y participan regularmente en torneos o competencias deportivas.

La edad de los participantes fluctuó entre los 18 y 36 años, con una media de 20,8 y una desviación estándar de 2,5 años. 
La muestra se obtuvo mediante un muestreo por conveniencia, tratando de abarcar la representatividad de los diferentes semestres de cada una de las licenciaturas que se ofrecen en la Facultad de Ciencias de la Cultura Física de la Universidad Autónoma de Chihuahua.

\section{Instrumento}

El Autoinforme de Barreras para la Práctica de Ejercicio Físico (ABPEF) de Niñerola, Capdevila \& Pintanel (2006) consta de 17 ítems, agrupados en cuatro factores (ansiedad física y social, fatiga o pereza, obligaciones y falta de tiempo e instalaciones), que se responden según una escala tipo Likert de 0 a 10 puntos, donde valores cercanos a 0 indican "una razón poco probable que me impide practicar ejercicio físico las próximas semanas", y valores cercanos a 10 indican una "razón muy probable que me impide practicar ejercicio físico". Para nuestro estudio se hicieron dos adaptaciones a la versión de Niñerola et al. (2006): (a) la primera consistió en cambiar algunos términos utilizados en los ítems de la versión original con el fin de emplear un lenguaje más adecuado al contexto de la cultura mexicana; (b) la segunda consistió en aplicar el instrumento por medio de una computadora (figura 1) permitiendo así el almacenamiento de los datos sin previa codificación, con mayor precisión y evitando errores.

\section{FIGURA 1}

Ejemplo de respuesta para los ítems del cuestionario

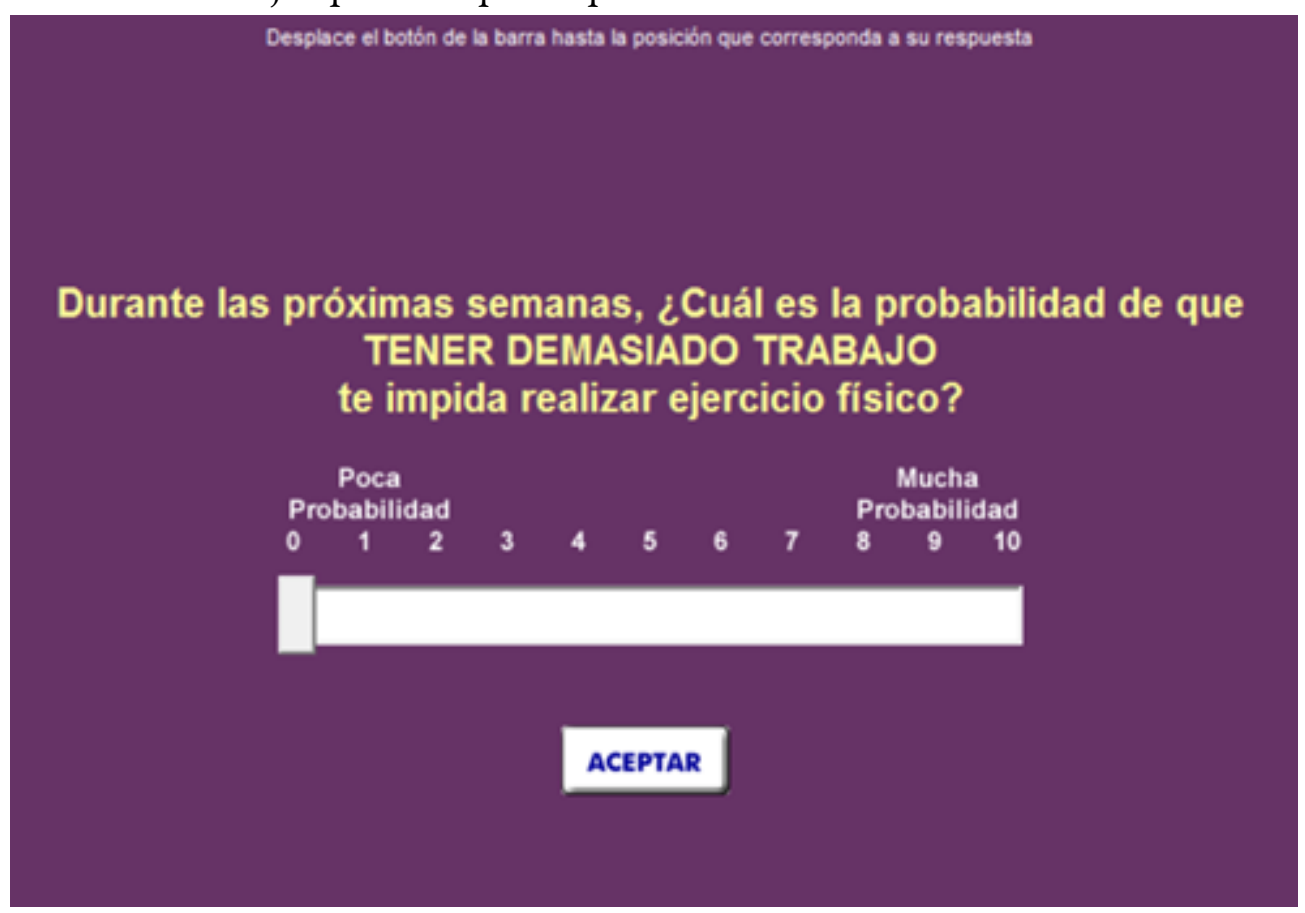

Fuente: elaboración propia

\section{Diseño}

En cuanto al diseño del estudio, se utilizó un enfoque cuantitativo con un diseño descriptivo y transversal tipo encuesta (Hernández, Fernández \& Baptista, 2014). La variable independiente fue la práctica deportiva (atletas y no atletas) y las variables dependientes el promedio de las puntuaciones obtenidas en cada una de las subescalas del cuestionario ABPEF. 


\section{Procedimiento}

Se invitó a participar en el estudio a los alumnos de las licenciaturas que se ofrecen en la Facultad de Ciencias de la Cultura Física (FCCF) de la Universidad Autónoma de Chihuahua.

Los que aceptaron participar firmaron la carta de aceptación correspondiente. Luego se aplicó el instrumento, antes descrito, por medio de una computadora personal (módulo administrador del instrumento del editor de escalas de ejecución típica), en una sesión de aproximadamente 20 minutos; en los laboratorios o centros de cómputo de la FCCF.

$\mathrm{Al}$ inicio de cada sesión se hizo una pequeña introducción sobre la importancia de la investigación y de cómo acceder al instrumento. Se les solicitó la máxima sinceridad y se les garantizó la confidencialidad de los datos que se obtuvieran. Las instrucciones de cómo responder se encontraban en las primeras pantallas; antes del primer reactivo del instrumento. Al término de la sesión se les agradeció su participación.

Una vez aplicado el instrumento se procedió a recopilar los resultados por medio del módulo generador de resultados del editor de escalas versión 2.0 (Blanco et al., 2013).

\section{Análisis de datos}

Se realizaron análisis estadísticos descriptivos (medias y desviaciones estándar) para todas las variables. Posteriormente, después de verificar que los datos cumplían con los supuestos de los análisis estadísticos paramétricos, se llevó a cabo un análisis multivariante de varianza (MANOVA), seguido por los análisis de varianza univariados (ANOVAs), para examinar las diferencias entre atletas y no atletas en cuanto a su percepción de barreras para la práctica de ejercicio físico. Por otra parte, el tamaño del efecto se estimó mediante el eta cuadrado $(\eta 2)$. Todos los análisis estadísticos se realizaron con el programa SPSS versión 21,0 para Windows. El nivel de significación estadística se estableció en $p=, 05$.

\section{Resultados}

La Tabla 1 muestra los valores de las medias y las desviaciones estándar de las puntuaciones de los cuatro factores estudiados sobre la percepción de barreras para la práctica de ejercicio físico, así como los resultados del MANOVA y los subsiguientes ANOVAs. Los resultados del MANOVA mostraron diferencias globales estadísticamente significativas entre atletas y no atletas en las puntuaciones promedio de los factores del Autoinforme de Barreras para la Práctica de Ejercicio Físico (Wilks' $\lambda=, 88 ; p<, 001 ; \eta 2=, 12$ ). Posteriormente, los resultados de los ANOVAs mostraron que los no atletas reportan mayor presencia de barreras en las cuatro dimensiones estudiadas; ansiedad física y social $(F=20,92, p<, 001)$, fatiga o pereza $(F$ $=88,36, p<, 001)$, obligaciones y falta de tiempo $(F=121,73, p<, 001)$ e instalaciones $(F=9,08, p<, 01)$. 
TABLA 1

Resultados MANOVA y ANOVAs para las diferencias en percepción de barreras para la práctica de ejercicio físico entre atletas y no atletas.

\begin{tabular}{|l|c|c|c|c|c|}
\hline & $\begin{array}{c}\text { No atletas } \\
(n=630)\end{array}$ & $\begin{array}{c}\text { Atletas } \\
(n=593)\end{array}$ & $F$ & $p$ & $\eta^{2}$ \\
\hline & & & 39,94 & $<, 001$ &, 12 \\
\hline Ansiedad fisica y social & $1,44(2,04)$ & $0,94(1,76)$ & 20,92 & $<, 001$ &, 02 \\
\hline$-y a t i g a$ o pereza & $2,95(1,97)$ & $1,94(1,78)$ & 88,36 & $<, 001$ &, 07 \\
\hline $\begin{array}{l}\text { Obligaciones y falta de } \\
\text { tiempo }\end{array}$ & $3,98(2,67)$ & $2,42(2,26)$ & 121,73 & $<, 001$ &, 09 \\
\hline \begin{tabular}{l} 
Instalaciones \\
\hline
\end{tabular} & $1,82(1,83)$ & $1,49(1,96)$ & 9,08 & $<, 01$ &, 01 \\
\hline
\end{tabular}

Nota Los valores descriptivos se presentan como media (desviación estándar)

\section{Discusión}

Los resultados obtenidos apuntan a los posibles beneficios que la práctica de actividad físico-deportiva misma puede generar menor presencia de barreras para su realización. Así pues, sería importante que se valorase la práctica de la actividad físico-deportiva como un factor positivo no solo para la salud, sino también como un elemento que en sí misma reduce la percepción de barreras para la práctica del ejercicio físico, ya que como refleja este estudio, podría potenciar efectos positivos que conllevan a una disminución de la percepción de barreras para su práctica; lo que concuerda con lo reportado en otras investigaciones donde se afirma que los sedentarios, en comparación con los activos físicamente de la misma edad y nivel de estudios, perciben en mayor medida que la falta de tiempo, la pereza o el estar cansados les impiden realizar actividad física (Gómez-López, Gallegos \& Extremera, 2010; Ramírez-Vélez, Triana-Reina, Carrillo \& Ramos-Sepúlveda, 2016; Rubio \& Varela, 2016). Sin embargo es sumamente importante determinar qué características (tipo, intensidad, frecuencia, etc.) y mediante qué mecanismos la práctica sistemática del ejercicio reduce la percepción de barreras para su realización.

\section{Conclusiones}

De los resultados mostrados, de su análisis y de su discusión, se pueden obtener las siguientes conclusiones:

1. Los universitarios que practican algún deporte y participan regularmente en torneos o competencias deportivas en relación a los que no lo hacen, perciben menor probabilidad en la presencia de barreras para la práctica de ejercicio físico, favoreciendo la práctica de actividad física en mayor medida que los no practicantes.

2. Las diferencias encontradas entre las poblaciones estudiadas en cuanto a la percepción de barreras para la práctica de ejercicio físico, sugieren además que al diseñar cualquier tipo de intervención que tenga como objetivo la disminución de la percepción de las mismas habrá que tomar en cuenta a la variable práctica deportiva; no obstante, es preciso desarrollar más investigación al respecto pues el tema trasciende del todo los alcances de la presente investigación.

Por último, cuando menos dos limitaciones están presentes en este trabajo. La primera es que los participantes son solo estudiantes universitarios mexicanos, lo que supone una amenaza para la posibilidad de generalizar estos resultados. Ampliar la muestra (agregando por ejemplo adultos jóvenes que no son 
estudiantes) es un área de trabajo de cara al futuro. La segunda limitación proviene del propio instrumento de medición, que se basa en el autoinforme y que por ello puede contener los sesgos que se derivan de la deseabilidad social.

\section{Agradecimientos}

Este estudio es parte de un proyecto financiado por la Secretaría de Educación Pública-Subsecretaría de Educación Superior-Dirección de Superación Académica- Programa para el Desarrollo Profesional Docente (DE-13-6894).

\section{REFERENCIAS BIBLIOGRÁFICAS}

Alley, T. (1991). Visual detection of body-weight change in young women. Perceptual and motor skills, 73(3), 904-906. Bibiloni, M. d. M. , Pich, J. , Córdova, A. , Pons, A. \& Tur, J. A. (2012). Association between sedentary behaviour and socioeconomic factors, diet and lifestyle among the Balearic Islands adolescents. BMC Public Health, 12(718), 1-11. doi: https://10.1186/1471-2458-12-718

Blanco, H. , Ornelas, M. , Tristán, J. L. , Cocca, A. , Mayorga-Vega, D. , López-Walle, J. \& Viciana, J. (2013). Editor for creating and applying computerise surveys. Procedia Social and Behavioral Sciences, 106, 935-940. doi: http s://10.1016/j.sbspro.2013.12.105

Camacho, M. J. , Fernández, E. \& Rodríguez, M. I. (2006). Imagen corporal y práctica de actividad física en las chicas adolescentes: incidencia de la modalidad deportiva. Revista Internacional de Ciencias del Deporte, 3(2), 1-19.

Cocca, A. , Liukkonen, J. , Mayorga-Vega, D. \& Viciana, J. (2014). Health-related physical activity levels in Spanish youth and young adults. Perceptual and motor skills, 118(1), 247-260.

Cuenca-García, M. , Ortega, F. B. , Ruiz, J. R. , González-Gross, M. , Labayen, I. , Jago, R., .. . Sjöström, M. (2014). Combined influence of healthy diet and active lifestyle on cardiovascular disease risk factors in adolescents. Scandinavian Journal of Medicine and Science in Sports, 24(3), 553-562. doi: https://10.1111/sms.12022

Gillis, L., Tomkinson, G. , Olds, T. , Moreira, C. , Christie, C. , Nigg, C., .. Willem, V. M. (2013). Research priorities for child and adolescent physical activity and sedentary behaviours: an international perspective using a twinpanel Delphi procedure. International Jorunal of Behavioral Nutrition and Physical Activity, 10(112), 1-8. doi: https://10.1186/1479-5868-10-112

Gómez-López, M. , Gallegos, A. G. \& Extremera, A. B. (2010). Perceived Barriers by University Students in the Practice of Physical Activities. Journal of Sports Science and Medicine, 9(3), 374-381.

Hernández, R. , Fernández, C. \& Baptista, P. (2014). Metodología de la investigación. México: McGraw- Hill.

Katzmarzyk, P. \& Janssen, I. (2003). Physical inactivity, excess adiposity and premature mortality. Obesity Reviews, 4(4), 257-290.

Kokkinos, P. (2012). Physical Activity, Health Benefits, and Mortality Risk. ISRN Cardiology, 2012, 1-14. doi: http s://10.5402/2012/71878

Longmuiremail, P., Colley, R., Wherley, V. \& Tremblay, M. (2014). Risks and benefits of childhood physical activity. The Lancet Diabets Endocrinologye Psychology, 2(11), 861-862. doi: https://10.1016/S2213-8587(14)70221-9

Martin, K. A. \& Bassett, R. L. (2012). Exercise and Changes in Body Image, Ginis. En T. F. Cash \& L. Smolak (Eds.), Body Image A Handbook of Science, Practice, and Prevention. New York: Guilford Press.

Niñerola, J. , Capdevila, L. \& Pintanel, M. (2006). Barreras percibidas y actividad física: el autoinforme de barreras para la práctica de ejercicio físico. Revista de Psicología del Deporte, 15(1), 53-69.

Ramírez-Vélez, R. , Triana-Reina, H. R. , Carrillo, H. A. \& Ramos-Sepúlveda, J. A. (2016). Percepción de barreras para la práctica de la actividad física y obesidad abdominal en universitarios de Colombia. Nutrición Hospitalaria, 33(6), 1317-1323. doi: https://10.20960/nh.777 
Reiner, M. , Niermann, C. , Jekauc, D. \& Wol, A. (2013). Long-term health benefits of physical activity - a systematic review of longitudinal studies. BMC Public Health, 13, 1-9. doi: https://10.1186/1471-2458-13-813

Rubio, R. F. \& Varela, M. T. (2016). Barreras percibidas en jóvenes universitarios para realizar actividad física. Revista Cubana de Salud Pública, 42(1), 61-69.

Serrano-Sanchez, J. A. , Martí-Trujillo, S. , Lera-Navarro, A. , Dorado-García, C. , González-Henríquez, J. J. \& SanchísMoysi, J. (2011). Associations between Screen Time and Physical Activity among Spanish Adolescents. Plos One, 6(9), 1-9. doi: https://10.1371/journal.pone.0024453

Steptoe, A., Wardle, J. , Cui, W. , Bellisle, F. , Zotti, A.-M. , Baranyai, R. \& Sanderman, R. (2002). Trends in Smoking, Diet, Physical Exercise, and Attitudes toward Health in European University Students from 13 Countries, 1990-2000. Preventive Medicine, 35, 97-104. doi: https://10.1006/pmed.2002.1048

Tornero, I. \& Sierras, Á. (2008). Satisfacción corporal y actividad fisica en el alumnado de la facultad de ciencias de la educación de la universidad de Huelva. Paper presented at the IV Congreso Internacional y XXV Nacional de Educación física, Córdoba, España.

Tucker, L. A. \& Mortell, R. (1993). Comparison of the effects of walking and weight training programs on body image in middle-aged women: An experimental study. American Journal of Healt Promotion, 8(1), 34-42.

Urrutia, S. , Azpillaga, I. , de Cos, G. L. \& Muñoz, D. (2010). Relación entre la percepción de estado de salud con la práctica físicodeportiva y la imagen corporal en adolescentes. Cuadernos de Psicología del Deporte, 20(2), 51-56.

Williams, P. A. \& Cash, T. F. (2001). Effect of a circuit weight training program on the body images of collage students. Internacional Journal of Eating Disorders, 30(1), 75-82. 